\title{
High correlation between skin color based on CIELAB color space, epidermal melanocyte ratio, and melanocyte melanin content
}

\author{
Wen-Shyan Huang ${ }^{1}$ ， Yi-Wen Wang ${ }^{2}$, Kun-Che Hung ${ }^{3}$ ， Pai-Shan Hsieh ${ }^{3}$, Keng-Yen Fu ${ }^{3}$, Lien-Guo Dai ${ }^{4}$, \\ Nien-Hsien Liou ${ }^{2}$, Kuo-Hsing Ma ${ }^{2}$, Jiang-Chuan Liu ${ }^{2}$, Niann-Tzyy Dai ${ }^{\text {Corresp. } 3}$ \\ 1 Division of Plastic and Reconstructive Surgery, Zuoying Branch of Kaohsiung Armed Forces General Hospital, Kaohsiung, Taiwan, R.O.C. \\ 2 Department of Biology and Anatomy, National Defense Medical Center, Taipei, Taiwan, R.O.C. \\ 3 Division of Plastic and Reconstructive Surgery, Department of Surgery, Tri-Service General Hospital, National Defense Medical Center, Taipei, Taiwan, \\ R.O.C. \\ 4 Department of Orthopedics, Shuang Ho Hospital, Taipei Medical University, Taipei, Taiwan, R.O.C. \\ Corresponding Author: Niann-Tzyy Dai \\ Email address: niantzyydai@gmail.com
}

Background. To treat skin color disorders, such as vitiligo or burns, melanocytes are transplanted for tissue regeneration. However, melanocyte distribution in the human body varies with age and location, making it difficult to select the optimal donor skin to achieve a desired color match. Determining the correlations with the desired skin color measurement based on CIELAB color, epidermal melanocyte numbers, and melanin content of individual melanocytes is critical for clinical application.

Method. Fifteen foreskin samples from Asian young adults were analyzed for skin color, melanocyte ratio (melanocyte proportion in the epidermis), and melanin concentration. Furthermore, an equation was developed based on CIELAB color with melanocyte ratio, melanin concentration, and the product of melanocyte ratio and melanin concentration. The equation was validated by seeding different ratios of keratinocytes and melanocytes in tissue-engineered skin substitutes, and the degree of fitness in expected skin color was confirmed.

Results. Linear regression analysis revealed a significant strong negative correlation $\left(r=-0.847, R^{2}=\right.$ 0.717 ) between CIELAB L* value and the product of the epidermal melanocyte ratio and cell-based melanin concentration. Furthermore, the results showed that an optimal skin color match was achieved by the formula.

Discussion. We found that $L^{*}$ value was correlated with the value obtained from multiplying the epidermal melanocyte ratio (R) and melanin content $(M)$ and that this correlation was more significant than either L* vs M or L* vs R. This suggests that more accurate prediction of skin color can be achieved by considering both $\mathrm{R}$ and $\mathrm{M}$. Therefore, precise skin color match in treating vitiligo or burn patients would be potentially achievable based on extensive collection of skin data from people of Asian descent. 
1 High correlation between skin color based on CIELAB color space, epidermal melanocyte

2 ratio, and melanocyte melanin content

3 Wen-Shyan Huang ${ }^{1}$, Yi-Wen Wang ${ }^{2}$, Kun-Che Hung ${ }^{3}$, Pai-Shan Hsieh ${ }^{3}$, Keng-Yen Fu ${ }^{3}$, Lien-

4 Guo Dai ${ }^{4}$, Nien-Hsien Liou ${ }^{2}$, Kuo-Hsing $\mathrm{Ma}^{2}$, Jiang-Chuan Liu ${ }^{2}$, Niann-Tzyy Dai ${ }^{3}$

$5 \quad{ }^{1}$ Division of Plastic and Reconstructive Surgery, Zuoying Branch of Kaohsiung Armed Forces

6 General Hospital, Kaohsiung, Taiwan, R.O.C.

$7 \quad 2$ Department of Biology and Anatomy, National Defense Medical Center, Taipei, Taiwan, 8 R.O.C.

$9{ }^{3}$ Division of Plastic and Reconstructive Surgery, Department of Surgery, Tri-Service General

10 Hospital, National Defense Medical Center, Taipei, Taiwan, R.O.C.

$11{ }^{4}$ Department of Orthopedics, Shuang Ho Hospital, Taipei Medical University, Taipei, Taiwan, 12 R.O.C.

13

14 Wen-Shyan Huang and Yi-Wen Wang were the first author and equal contribution in this

15 manuscript.

\section{Corresponding author}

$17 *$ Niann-Tzyy Dai

18 Division of Plastic and Reconstructive Surgery, Department of Surgery, Tri-Service General 19 Hospital, National Defense Medical Center, Taiwan, R.O.C.

20 No. 325, Section 2, Cheng-Kung Road, Nei-Hu District, Taipei 114, Taiwan, R.O.C.

21 E-mail: niantzyydai@gmail.com 


\section{ABSTRACT}

26 Background. To treat skin color disorders, such as vitiligo or burns, melanocytes are transplanted

27 for tissue regeneration. However, melanocyte distribution in the human body varies with age and 28 location, making it difficult to select the optimal donor skin to achieve a desired color match.

29 Determining the correlations with the desired skin color measurement based on CIELAB color, 30 epidermal melanocyte numbers, and melanin content of individual melanocytes is critical for 31 clinical application.

32 Method. Fifteen foreskin samples from Asian young adults were analyzed for skin color, 33 melanocyte ratio (melanocyte proportion in the epidermis), and melanin concentration.

34 Furthermore, an equation was developed based on CIELAB color with melanocyte ratio, melanin 35 concentration, and the product of melanocyte ratio and melanin concentration. The equation was 36 validated by seeding different ratios of keratinocytes and melanocytes in tissue-engineered skin 37 substitutes, and the degree of fitness in expected skin color was confirmed.

38 Results. Linear regression analysis revealed a significant strong negative correlation $(r=-0.847$, $\left.39 \mathrm{R}^{2}=0.717\right)$ between CIELAB L* value and the product of the epidermal melanocyte ratio and 40 cell-based melanin concentration. Furthermore, the results showed that an optimal skin color 41 match was achieved by the formula.

42 Discussion. We found that $\mathrm{L}^{*}$ value was correlated with the value obtained from multiplying the 43 epidermal melanocyte ratio $(\mathrm{R})$ and melanin content $(\mathrm{M})$ and that this correlation was more 44 significant than either $\mathrm{L}^{*}$ vs $\mathrm{M}$ or $\mathrm{L}^{*}$ vs $\mathrm{R}$. This suggests that more accurate prediction of skin 45 color can be achieved by considering both $\mathrm{R}$ and $\mathrm{M}$. Therefore, precise skin color match in treating 46 vitiligo or burn patients would be potentially achievable based on extensive collection of skin data 47 from people of Asian descent. 
49 INTRODUCTION

50 Evaluating skin color match for treating skin color disorder requires an objective

51 measurement to quantify visual skin color into numerous levels. The principles of color

52 measurement established by the Commission International d'Eclairage (CIE) have been widely

53 applied to skin. Color values have been obtained by using reflectance spectroscopy, expressed in

54 terms of color space $L^{*}$ value, hue angle, and chroma values (Weatherall \& Coombs, 1992).

55 Differences of trichromatic color vision between individuals are identified in terms of one, two, or

56 all three CIE color-space parameters: $\mathrm{L}^{*}$ value, $\mathrm{a}^{*}$ value, and $\mathrm{b}^{*}$ value (CIELAB). The $\mathrm{L}^{*}$ value,

57 which correlates to perceived lightness and ranges from absolute black (0) to absolute white

$58(+100)$, is the most sensitive of the trichromatic values to skin color change. Because the method

59 is quantitative and the principles are internationally recognized, these color-space parameters are

60 proposed for the unambiguous communication of skin color information that relates directly to

61 visual observations of clinical importance or scientific interest (Stevenson et al., 2012).

62 Medical therapy for hypopigmentation disorders has improved in recent years; however,

63 complete repigmentation and perfect skin color match seem to be unsatisfactory in most patients

64 treated (Iannella et al., 2016). Therefore, a variety of surgical grafting techniques have been

65 performed to treat skin color disorders that do not respond to medical treatment, such as split

66 thickness grafts, cultured autologous melanocytes, minigrafts, suction blister grafts, and non-

67 cultured epidermal suspension, among others (Gauthier \& Benzekri, 2012). These techniques

68 contain different advantages and disadvantages with respect to cost, time consumption, treatment

69 area and location, need for equipment, and possible outcome of abnormal appearance (Gauthier \&

70 Benzekri, 2012). A previous study reported that cultured skin substitutes (CSS) fabricated from

71 autologous keratinocytes and fibroblasts seeded onto collagen-glycosaminoglycan substrates

72 could be applied to excised, full-thickness burns on 5 patients. Spontaneous repigmentation of CSS 
73 treatment from passenger melanocytes in keratinocyte culture was found within 2 months after 74 grafting (Harriger et al., 1995). Kahn \& Cohen (1995) used very thin epithelial sheet grafts

75 harvested by dermatome from pigmented donor areas and then covered it with petrolatum gauze

76 on five patients with stable vitiligo, which all resulted in excellent repigmentation and no scarring

77 developed. Gupta et al (1999) collected the donor epidermal sheets from the blisters developed

78 through the cutaneous suction apparatus, and then grafted onto the denuded skin lesion site.

79 Alternatively, Falabella (2001) implanted very small dermo-epidermal grafts on recipient sites

80 prepared with minipunches of similar size. Mulekar (2003) and van Geel et al (2001) both grafted

81 non-cultured melanocyte-keratinocyte suspensions onto previously dermabraded vitiligo lesions

82 and achieved a high repigmentation. Transplantation of pure primary melanocyte cultures has also

83 been proposed with a better response, relatively homogeneous skin color, and capability for use

84 on larger lesion areas (Chen et al., 2004; Kaufmann et al., 1998; Lontz et al., 1994; Olsson \&

85 Juhlin, 2002).

86 Currently, the outcomes of repigmentation through pigment skin tissue engineering or

87 pigment cell transfer are still unpredictable. Previous studies indicated that regulating cutaneous

88 pigmentation in cultured skin substitutes was feasible by titration of human melanocytes and

89 keratinocytes (Swope, Supp \& Boyce, 2002; Swope et al., 1997). However, epithelial melanocytes

90 used in Swope's experiments came from a single donation; thus, the influence of different sources

91 on skin pigmentation could not be determined. The aim of this work was to identify how

92 melanocyte numbers and activity from different Asian people modulate skin color via a well-

93 defined correlation between skin color and melanocytes. A total of 15 human adult foreskin

94 samples donated from Asian individuals were analyzed and the correlations among skin color

95 determined by CIELAB color-space parameter: $\mathrm{L}^{*}$ value, epidermal melanocyte ratio, and melanin 
96 content per $10^{6}$ melanocytes was investigated. Finally, we evaluated the feasibility of applying this

97 skin color relationship in skin tissue engineering.

98

99 MATERIALS AND METHODS

\section{Materials}

Gibco Company (New York, USA) supplied Trypsin-EDTA solution (10x) and Penicillinsaline). Melanocyte culture medium contains Medium 254 (Cascade Biologics Inc., Oregon, Portland), 1\% Human Melanocyte Growth Supplement (HMGS, Cascade Biologics Inc., Oregon, (DCM) was purchased from J. T. Baker (Phillipsburg, USA).

\section{Inclusion criteria for skin samples collection}

114 Board (IRB) in the Tri-Service General Hospital, R.O.C. (TSGHIRB No.: 095-05-0068). Then the written informed consent was obtained from each donor.

\section{Primary culture of human epidermal keratinocytes and melanocytes}

118 from equal size $(1 \mathrm{~cm} \times 1 \mathrm{~cm}$ in square $)$ of human young adult foreskin samples obtained in the 
119 surgery of circumcision. For culture of PHEKs, the foreskin sample was initially immersed in 10

$120 \mathrm{ml}$ of $0.2 \%$ Dispase II solution (Sigma, St. Louis, USA) at $4^{\circ} \mathrm{C}$ for 48 hours and diced in pieces,

121 followed by incubation in $0.05 \%$ Trypsin-EDTA solution for $15 \mathrm{~min}$. The pelleted cells were

122 obtained by a centrifugation at $1300 \mathrm{rpm}$ for $5 \mathrm{~min}$, seeded in a fibronectin/collagen (AthenaES,

123 Maryland, USA) coated flask and cultured in EpiLife ${ }^{\circledR}$ keratinocyte medium at $37^{\circ} \mathrm{C}$ in $5 \% \mathrm{CO}_{2}$.

124 For primary culture of PHEMs, the epidermal cell cultured in the EpiLife ${ }^{\circledR}$ medium were

125 transferred to melanocyte culture medium after the epidermal primary culture and incubated at

$12637^{\circ} \mathrm{C}$ in $5 \% \mathrm{CO}_{2}$. Highly selected culture of PHEMs was then obtained after passage 2.

\section{Skin color measurement}

128 Foreskin samples were obtained immediately after surgery, blood and adipose tissues were

129 removed, and color was measured in triplicate for each sample ex vivo using a color reader CR-10

130 (Konica Minolta, Osaka, Japan). The color differences were displayed in terms of trichromatic L*,

$131 \mathrm{a}^{*}$, and $\mathrm{b}^{*}$ values as determined by the CIE. Since the $\mathrm{L}^{*}$ value correlates to perceived color

132 brightness (black vs. white) and is the most sensitive trichromatic value for measuring skin

133 pigmentation in the pilot study, it was chosen rather than $\mathrm{a}^{*}$ and $\mathrm{b}^{*}$ values to represent measured

134 skin color in this study. The $\mathrm{L}^{*}$ value was measured in triplicate by detecting the top surface of

135 the collected foreskin sample and recorded as mean $\pm \mathrm{SE}$ (standard error).

136 Analysis of melanocyte ratio in epidermal cells

137 Fluorescent-activated cell sorting (FACS) was used to distinguish melanocytes from 138 epidermal cells. Epidermal cell suspensions of foreskin were immediately prepared after surgery,

139 which were then centrifuged at $300 \times g$ for $5 \mathrm{~min}$ and the cell pellets were treated with a 140 Cytofix/CytoPerm Plus kit (BD, New Jersey, USA) for subsequent flow cytometry. Briefly,

141 melanocytes were isolated from epidermal cells by adherence. Next, the cells were permeabilized 
142 with $200 \mu \mathrm{l} \mathrm{Cytofix} / \mathrm{CytoPerm}$ solution for $20 \mathrm{~min}$ at $4^{\circ} \mathrm{C}$ and washed with $1 \mathrm{ml}$ Perm/Wash Buffer

143 (BD, New Jersey, USA) twice. For labeling melanocytes, permeabilized cells were stained with

144 mouse anti-human melan-A IgG (Santa Cruz Biotechnology, California, USA) and incubated with

145 fluorescein isothiocyanate-conjugated (FITC) goat anti-mouse IgG (Jackson, Pennsylvania, USA).

146 Negative controls for melan-A staining consisted of cells stained with FITC goat anti-mouse IgG

147 only. The samples were then analyzed with five replicates being used for each sample, the samples

148 were analyzed on a FACS Calibur flow cytometer using CellQuest software (BD, New Jersey,

149 USA); the mean value was then obtained.

150 Determination of melanin concentration

151 The melanin production of melanocytes from distinct foreskin samples in term of total

152 melanin content per $10^{6}$ melanocytes was measured by a spectrophotometric assay. Briefly,

153 Purified primary human epidermal melanocytes (PHEMs) $\left(10^{6}\right.$ cells per pellet) were lysed with 1

$154 \mathrm{M} \mathrm{NaOH}$ at $80^{\circ} \mathrm{C}$ for $2 \mathrm{~h}$. After centrifugation at $12,000 \times g$ for $10 \mathrm{~min}$ at room temperature, the

155 supernatants were transferred to fresh tubes and melanin content was determined in triplicate for 156 each sample by measuring the absorbance at $490 \mathrm{~nm}$ in a spectrophotometer and expressed as

157 microgram of melanin per $10^{6}$ cells. Synthetic melanin (Sigma, St. Louis, USA) was used to plot 158 a standard curve.

159 Preparation of collagen/PCL membranous scaffolds

160 Collagen/polycaprolactone (PCL) scaffolds were prepared as described previously (Dai et al. 161 2004). Briefly, type I collagen was dissolved in $1 \%$ acetic acid, generating a $0.25 \% \mathrm{w} / \mathrm{v}$ collagen 162 solution. The collagen solution was poured into a round glass vial (diameter $2.5 \mathrm{~cm}$ and height 4.5 $163 \mathrm{~cm}$ ) and frozen at $-20^{\circ} \mathrm{C}$ for 50 minutes, followed by lyophilizing in a freeze-dryer (DRC-1100,

164 Eyela, Japan) for 24 hours. The PCL/dichloromethane (DCM) solution $(2.5 \% \mathrm{w} / \mathrm{v})$ was then added 
165 to the freeze-dried collagen matrix to prepare a 1:20 w/w collagen/PCL scaffold. The glass vial

166 was kept open overnight to allow DCM evaporation.

167 Color measurement of pigmented tissue-engineered skin substitutes based on collagen/PCL

168 scaffold

169 Pigmented tissue engineered skin substitutes (diameter $2.5 \mathrm{~cm}$ ) with primary human 170 epidermal keratinocytes (PHEKs) and/or PHEMs (total cell density of $4.72 \times 10^{4} \mathrm{cells} / \mathrm{cm}^{2}$ ) in

171 various ratios were prepared based on the $\mathrm{L}^{*}$ value of calculated skin color relationship including

172 L40 (melanocyte ratio was 1.79), and L50 (melanocyte ratio was 4.83) (L* values as 40 and 50

173 respectively) groups. Epilife serum-free medium was used to incubate the skin substitute at $37^{\circ} \mathrm{C}$

174 in $5 \% \mathrm{CO}_{2}$. The 6-well tissue culture plastics without cells served as the blank group. The color of

175 pigmented tissue-engineered skin substitutes was measured with a color reader at various time

176 points $(10,20,30,40,50,60$, and 70 days $)$.

177 The analysis of correlation for skin color and melanocytes

178 To estimate the relationships of epidermal melanocyte ratio or melanin concentration vs skin 179 lightness based on foreskin CIELAB L* value, linear regression analysis was performed. The 180 coefficient of determination $\left(\mathrm{R}^{2}\right)$ and Pearson's correlation coefficient $(\mathrm{r})$ were calculated to

181 measure goodness of fit of a statistical model and the strength and direction of the linear 182 relationship.

183 Statistical analysis

184 The continuous data such as $\mathrm{L}^{*}$ value of foreskin samples and tissue-engineered skin 185 substitutes, melanin amount, and the melanocyte ratio in epidermal cells were shown as "mean \pm 186 SD (SD: standard deviation)". The variables were grouped first, comparing mean values in 187 categories, Pearson's correlation coefficient (r) and coefficient of determination consecutively. 
188 Simple linear regression analysis was used afterwards to evaluate the combined effect of several 189 variables and to estimate the coefficient of determination. Statistical Product and Service Solutions 190 (SPSS) was used to perform a stepwise forward selection procedure. Thus, for each iterative loop 191 of this procedure one more variable was integrated into the new formula. All statistical result is 192 statistically significant when the $P$ value is less than $0.05(P<0.05)$. Statistical analysis was 193 performed using Statistical Package for the Social Sciences, Version 12.0 (SPSS Inc., Chicago, 194 Illinois).

195

196

197

198

199

200

201

202

203

204

205

206

207

208

209

210

\section{RESULTS}

\section{Measurement of skin color ( $L^{*}$ value)}

The study design of measuring skin color of foreskin samples, melanin concentration, and epidermal melanocyte ratios for establishing a relationship with skin color is shown in Fig. 1. The skin color of 15 human young adult foreskin samples was measured immediately after surgery by a color reader ex vivo yielding values from $39.43 \pm 0.21$ to $52.37 \pm 1.90$ as determined by CIELAB color-space parameter: L* value (Table 1).

\section{Melanocyte ratio in epidermal cells $(R)$}

The concentration of melanocytes in foreskin epidermal cell suspensions was measured using FACS. The results showed ratios of melanocytes to epidermal cells ranging from $1.40 \pm 0.28$ to $3.58 \pm 0.07 \%$ (Table 1$)$

\section{Epidermal melanin production (M)}

To determine the melanin productivity of melanocyte isolated form each foreskin, PHEMs were cultured for 2 passages reaching a purity of $99.4 \%$ and analyzed using immunohistochemistry 
211 assays and FACS (Fig. 2). The results showed that the melanin production of 15 PHEMs ranged

212 from $17.99 \pm 0.50$ to $81.91 \pm 1.92 \mu \mathrm{g} / 10^{6}$ cells (Table 1$)$.

213

\section{The analysis of correlation for skin color}

215 We next examined the correlation between skin color and melanin concentration. Plots of L*

216 value against epidermal melanocyte ratio and $\mathrm{L}^{*}$ value against melanin concentration per $10^{6}$

217 melanocytes were generated based on the data shown in Table 1 (Fig. 3). The results revealed no

218 correlation between $\mathrm{L}^{*}$ value and epidermal melanocyte ratio $\left(\mathrm{r}=-0.081, \mathrm{R}^{2}=0.0066\right)$. However,

$219 \mathrm{~L}^{*}$ value was negatively correlated with melanin concentration $\left(\mathrm{r}=-0.592, \mathrm{R}^{2}=0.3503\right)(P=0.02)$

220 (Fig. 3). However, there were significant associations between the $\mathrm{L}^{*}$ value with the production

221 of epidermal melanocyte ratio and melanin content. A further linear regression analysis showed a

222 significantly strong negative correlation $\left(r=-0.847, \mathrm{R}^{2}=0.717\right)$ between $\mathrm{L}^{*}$ value and the

223 epidermal melanocyte ratio multiplied by cell-based melanin concentration (Fig. 4). Given these

224 results, an equation describing skin color relationship was generated: as $\mathrm{L}^{*}=\mathrm{a} \times(\mathrm{M} \times \mathrm{R})+\mathrm{b}(\mathrm{M}$ :

225 melanin concentration per $10^{6}$ melanocytes; R: epidermal melanocyte ratio; $\mathrm{a}=-0.095$; and $\mathrm{b}=$ 226 55.872).

227

228

Application of the skin color relationship in skin tissue engineering

229 As shown in Figure 5a, collagen/PCL constructs at 70 days were ranked in order of different 230 shades of black as PHEMs $>$ L40 $>$ L50 $>$ PHEKs $>$ Blank. The result of the L* value profile of 231 the collagen/PCL constructs is shown in Fig. 5 (b). Obvious changes of the L* values were noted 232 in 20 days post-culture for all groups except the blank control group. After 70 days, the $L^{*}$ value 233 of L40, and L50 groups were 43.50 \pm 1.87 , and 50.67 \pm 0.55 , respectively, whereas PHEMs and 
234 PHEKs exhibited values of $30.30 \pm 0.56$ and $79.73 \pm 0.60$, respectively.

235

236

237

238

239

240

241

242

243

244

245

246

247

248

249

250

251

252

253

254

255

256

\section{DISCUSSION}

To date, there is no method to accurately estimate transplanted skin pigments via a skin color formula used to fabricate tissue-engineered skin. Numerous previous studies have shown that skin constitutive pigmentation is determined by melanin production levels. For example, Alaluf et al. (2002b) performed correlation analysis and found the best correlation between the L* value and total melanin content in the epidermis. Del Bino et al. (2015) reported that total melanin content, including eumelanin and pheomelanin content, determines the constitutive skin pigmentation. Wakamatsu et al. (2006) indicated that cell-based melanin production and the predominant biological forms of melanin produced by melanocytes affect skin pigmentation. Additionally, implanting different numbers of melanocytes influences skin color. Swope et al. (2012) implanted $1.1 \times 10^{2}, 1.1 \times 10^{3}$, and $1.1 \times 10^{4}$ human melanocytes $/ \mathrm{cm}^{2}$ into athymic mice and found that mice with the highest density of melanocytes were significantly darker than mice in the other groups. Duval et al. (2014) confirmed that dermal fibroblasts influence the degree of skin pigmentation by measuring quantitative parameters related to skin color, melanin content, and melanocyte numbers in an in vitro skin system. Brankov et al. (2016) found that pigmented basal cell carcinomas (BCCs) have a higher mean melanocyte count compared to non-pigmented BCCs, indicating that the pigment is increased not only because of increased melanin, but also because of increased melanocyte counts. The density of melanocytes varies with the body site, with approximately 900 melanocytes per square $\mathrm{mm}$ on the back and approximately 1500 melanocytes per square $\mathrm{mm}$ in the genital region (Thingnes et al., 2012). Therefore, for the practical treatment of patients with skin color disorder, the implanted skin should be evaluated for both melanin production and 
257 melanocyte count from the donor site for precise color matching. We predict that regulating skin

258 color can be determined by native cell-based melanin production $(\mathrm{M})$ and melanocyte numbers in

259 the epidermis $(\mathrm{R})$. We found that the $\mathrm{L}^{*}$ value was correlated with the value of the multiplicative

260 product of the epidermal melanocyte ratio $(\mathrm{R})$ and melanin content $(\mathrm{M})$, and these values were

261 much higher than those of the two aforementioned parameters individually, suggesting that

262 considering the epidermal melanocyte ratio and melanin content strengthens the prediction of skin

263 color. Moreover, we performed statistical analysis and found a negative correlation between M

264 and $\mathrm{R}(\mathrm{r}=-0.5359, \mathrm{P}=0.04)$. On normalizing $\mathrm{M}$ to $\mathrm{R}(\mathrm{M} / \mathrm{R}) \mathrm{vs} \mathrm{L}^{*}$, a low correlation $(\mathrm{r}=0.3340$,

$265 \mathrm{P}=0.22$ ) was observed. This result also agreed with the result that the product of $\mathrm{M}$ and $\mathrm{R}$ is close

266 to a fixed value.

267 Although skin pigmentation is known to be regulated by melanocytes, the factors affecting 268 and regulating ethnic skin color are further to be determined (Hoath \& Leahy, 2003; Snell \& 269 Bischitz, 1963). Based on our previous research, skin color is essentially affected by the ethnicity 270 of the individual, while melanocyte density and differentiation are also influenced by the 271 environmental factors, such as ultraviolet radiation (UVR) and factors secreted by neighboring 272 keratinocytes and fibroblasts (Dai et al., 2018). The melanocortin 1 receptor (MC1R), a G protein273 coupled receptor that regulates the quantity and quality of melanin production, is the major 274 determinant of the pigment phenotype of the skin. Three agonists, such as alpha-melanocyte 275 stimulating hormone, adrenocorticotrophic hormone, and proopiomelanocortin, can activate $276 \mathrm{MC1R}$ via the cyclase/cAMP/protein kinase A signaling pathway. Next, the cAMP response 277 element binding protein is phosphorylated, resulting in the transcriptional induction of 278 microphthalmia-associated transcription factor (MITF). MITF is involved in regulating the 279 expression of melanogenic proteins, such as tyrosinase (TYR), tyrosinase-related protein 1, and 
280 tyrosinase-related protein 2 to regulate skin color (Gillbro \& Olsson, 2011). MITF activity can

281 also be regulated by different transcription factors or mediators secreted by keratinocytes and

282 fibroblasts, such as basic fibroblast growth factor, stem cell factor, endothelin-1, prostaglandins,

283 and leukotrienes. Based on a genome-wide association study, polymorphisms in three genes,

284 including SLC24A5, TYR, and SLC45A2, showed highly significant associations with melanin

285 content in the skin (Stokowski et al., 2007; Wilson et al., 2013). The SLC24A5 gene encoding the

286 NCKX5 protein, as a potassium-dependent sodium-calcium exchanger, exhibits lower exchange

287 activity, resulting in reduced melanogenesis and lighter skin in individuals (Wilson et al., 2013).

288 These regulatory genes in melanocytes are suggested to differ from race to race or even among

289 individuals and lead to different melanin production capabilities (Han et al. 2006). Alaluf et al

290 (2002a) studied the ethnic variation of melanin content and composition in the human skin from

291 photoprotected and photoexposed areas on human bodies. They found that melanosome size plays

292 a significant role in the variation of different ethnic skin types; African skin had the largest

293 melanosomes followed in turn by Indian, Mexican, Chinese, and European. In addition, the levels

294 of light-colored, alkali-soluble melanin including pheomelanin and DHICA-enriched eumelanin

295 in photoprotected skin areas for European, Chinese, and African skin are estimated as 43.2, 34.4,

296 and $15.1 \%$, respectively (Alaluf et al., 2002a).

297 In our study, L* value was correlated with melanin content but not epidermal melanocyte 298 ratio. A similar density of pigment-producing melanocytes in the skin $\left(\sim 1000 / \mathrm{mm}^{2}\right)$ has been 299 found in skin types from different races (Staricco \& Pinkus, 1957). However, the skin color of 300 people from the same group may vary because of different living habits, such as the time spent 301 outdoors, diet, or the use of sunscreen, among other factors. Taiwanese people comprise a multi302 racial population, including aborigines and immigrants from the mainland. This area was colonized 
303 by the Dutch and Japanese in the past. Therefore, the skin color may reflect different racial 304 characteristics showing high variation in skin samples in this study. In fact, there are few more 305 crucial factors affecting final skin color, including the amount of melanin, melanin composition, 306 and melanosome size across skin from a range of ethnicities. In addition, the higher level of 307 melanin production in darker skin was due to the continuously higher level of tyrosinase activity 308 in melanocytes. Taken together, the amount of melanin in the epidermis plays an important role in 309 skin color of individuals from different races, which agrees with our result that melanin content 310 was significantly correlated with $L^{*}$ value of CIELAB color space.

311 Given that the native melanin-producing capability of a melanocyte depends on genetic

312 factors (Fitzpatrick, Miyamoto \& Ishikawa, 1967; Szabo et al., 1969; Wakamatsu et al., 2006), in 313 clinical applications for autogenous pigment cell therapy, the cell-based melanin content is 314 constant in the same individual, and the predicted skin color matches represented as $\mathrm{L}^{*}$ value could 315 be detected from the normal skin area adjacent to the hypopigmented lesion site. Thus, a 316 meticulously estimated epidermal melanocyte ratio for the purpose of autogenous pigment cell

317 therapy applications would be attained based on the color formula, which presents as $L^{*}=0.095$ $318 \times(\mathrm{M} \times \mathrm{R})+55.872\left(\mathrm{M}:\right.$ melanin concentration per $10^{6}$ melanocytes; $\mathrm{R}:$ epidermal melanocyte 319 ratio). Moreover, when culturing various melanocyte ratios in the collagen/PCL scaffolds, the 320 results from in vitro experiments confirmed that the use of this skin color formula is feasible. The $321 \mathrm{~L}^{*}$ value of L40 and L50 groups was $43.50 \pm 1.87$ and $50.67 \pm 0.55$ respectively, very near the

322 predicted value. Therefore, predicting skin color is possible via collecting skin data for establishing

323 the skin color formula. In the clinic, the product of melanin production and melanocyte distribution

$324(\mathrm{M} \times \mathrm{R})$ is indicative of the darkness of skin color. The density of melanocytes differs in different 325 parts of the body. For skin autograft, a plastic surgeon often obtains skin grafts from different body 
326 sites for implantation at the recipient site. Based on the skin color formula derived in our study,

327 we can more precisely predict skin color by measuring melanin production by the donor skin and

328 adjusting the ratio of melanocytes and keratinocytes to produce a specific color in transplanted

329 skin to treat patients with skin color disorders, such as vitiligo or burn. The cell sheet technique or

330 plasma gel can be used, adjusting the ratio of melanocytes and keratinocytes to prepare

331 transplanted skins suitable for different skin color lesions.

332 The skin color formula still has some limitations that should be addressed before its clinical

333 application. Swope et al. found that cutaneous pigments would be present as a function of

334 melanocyte density and time after grafting due to the depletion of human melanocytes in CSS in

335 an animal model (Swope et al., 1997; Swope, Supp \& Boyce, 2002). Furthermore, skin color is

336 suggested to be determined by several other factors including the total quantity of melanin, the

337 proportion between the brown-black eumelanin and the yellow-red pheomelanin, and its

338 distribution involved in the epidermis (Naysmith et al., 2004). The $\log _{\mathrm{e}}$ values of

339 eumelanin/pheomelanin ratio were inversely related to the color variables b* (yellow-

340 blue $)\left(\mathrm{R}^{2}=0.51, P<0.001\right)$, a*(red-green $)\left(\mathrm{R}^{2}=0.47, P<0.001\right)$, and to a lesser extent $\mathrm{L}^{*}$ values

$341\left(\mathrm{R}^{2}=0.22, P<0.001\right)$ (Naysmith et al., 2004). On the other hand, three of cell types including

342 melanocytes, keratinocytes, and fibroblasts actively participate in regulating skin pigmentation

343 through secreted factors and their receptors, and their interactions may determine skin

344 pigmentation (Yin et al., 2014). The other chromophores present in the skin include

345 oxyhemoglobin, reduced hemoglobin, and carotene, which may influence the true skin color. Skin

346 blood flow increases with increases in hemoglobin (Tsuchida, Fukuda \& Kamata, 1991), and a*

347 values correlate linearly well with hemoglobin levels. The a* value indicates the redness of the

348 skin color and is mainly influenced by the degree of vascularization and the stretching of the skin

349 over surrounding tissues. Therefore, all three CIE color-space parameters: L* value, a* value, and 
$350 \mathrm{~b}^{*}$ value should be discussed in the future for perfect match of the skin color used in clinical 351 application.

352

353 CONCLUSIONS

354 A formula for estimating skin color based on CIELAB L* value, epidermal melanocyte ratio, 355 and melanin concentration was generated from people of Asian descent. This skin color formula 356 may serve as a useful methodology for determining distinct pigment cell concentrations for cell

357 therapy or for developing a pigmented skin tissue engineering model for transplantation and 358 pharmaceutical screening applications in vitro.

359 
360

361

362

363

364

365

366

367

368

369

370

371

372

373

374

375

376

377

378

379

380

381

382

383

384

385

386

387

388

389

390

391

392

393

394

395

\section{REFERENCES}

Alaluf S, Atkins D, Barrett K, Blount M, Carter N, and Heath A. 2002a. Ethnic variation in melanin content and composition in photoexposed and photoprotected human skin. Pigment Cell Research 15:112-118.

Alaluf S, Atkins D, Barrett K, Blount M, Carter N, and Heath A. 2002b. The impact of epidermal melanin on objective measurements of human skin colour. Pigment Cell Res 15:119-126.

Brankov N, Prodanovic EM, and Hurley MY. 2016. Pigmented basal cell carcinoma: increased melanin or increased melanocytes? J Cutan Pathol 43:1139-1142. 10.1111/cup.12819

Chen YF, Yang PY, Hu DN, Kuo FS, Hung CS, and Hung CM. 2004. Treatment of vitiligo by transplantation of cultured pure melanocyte suspension: analysis of 120 cases. Journal of the American Academy of Dermatology 51:68-74. 10.1016/j.jaad.2003.12.013

Dai NT, Williamson MR, Khammo N, Adams EF, and Coombes AG. 2004. Composite cell support membranes based on collagen and polycaprolactone for tissue engineering of skin. Biomaterials 25:4263-4271. 10.1016/j.biomaterials.2003.11.022

Del Bino S, Ito S, Sok J, Nakanishi Y, Bastien P, Wakamatsu K, and Bernerd F. 2015. Chemical analysis of constitutive pigmentation of human epidermis reveals constant eumelanin to pheomelanin ratio. Pigment Cell Melanoma Res 28:707-717. 10.1111/pcmr.12410.

Duval C, Cohen C, Chagnoleau C, Flouret V, Bourreau E, and Bernerd F. 2014. Key regulatory role of dermal fibroblasts in pigmentation as demonstrated using a reconstructed skin model: impact of photo-aging. PLoS One 9:e114182. 10.1371/journal.pone.0114182.

Falabella R. 2001. Surgical therapies for vitiligo and other leukodermas, part 1: minigrafting and suction epidermal grafting. Dermatologic Therapy 14:7-14. 10.1046/j.15298019.2001.014001007.x

Fitzpatrick TB, Miyamoto M, and Ishikawa K. 1967. The evolution of concepts of melanin biology. Archives of Dermatology 96:305-323.

Gauthier Y, and Benzekri L. 2012. Non-cultured epidermal suspension in vitiligo: from laboratory to clinic. Indian Journal of Dermatology, Venereology and Leprology 78:59-63. 10.4103/0378-6323.90947

Gillbro JM, and Olsson MJ. 2011. The melanogenesis and mechanisms of skin-lightening agents-existing and new approaches. Int J Cosmet Sci 33:210-221. 10.1111/j.14682494.2010.00616.x

Gupta S, Shroff S, and Gupta S. 1999. Modified technique of suction blistering for epidermal grafting in vitiligo. International Journal of Dermatology 38:306-309.

Han K, Choi T, and Son D. 2006. Skin color of Koreans: statistical evaluation of affecting factors. Skin Res Technol 12:170-177. 10.1111/j.0909-752X.2006.00145.x

Harriger MD, Warden GD, Greenhalgh DG, Kagan RJ, and Boyce ST. 1995. Pigmentation and 
396

397

398

399

400

401

402

403

404

405

406

407

408

409

410

411

412

413

414

415

416

417

418

419

420

421

422

423

424

425

426

427

428

429

430

431

microanatomy of skin regenerated from composite grafts of cultured cells and biopolymers applied to full-thickness burn wounds. Transplantation 59:702-707.

Hoath SB, and Leahy DG. 2003. The organization of human epidermis: functional epidermal units and phi proportionality. Journal of Investigative Dermatology 121:1440-1446. 10.1046/j.1523-1747.2003.12606.x

Iannella G, Greco A, Didona D, Didona B, Granata G, Manno A, Pasquariello B, and Magliulo G. 2016. Vitiligo: Pathogenesis, clinical variants and treatment approaches. Autoimmun Rev 15:335-343. 10.1016/j.autrev.2015.12.006

Kahn AM, and Cohen MJ. 1995. Vitiligo: treatment by dermabrasion and epithelial sheet grafting. Journal of the American Academy of Dermatology 33:646-648.

Kaufmann R, Greiner D, Kippenberger S, and Bernd A. 1998. Grafting of in vitro cultured melanocytes onto laser-ablated lesions in vitiligo. Acta Dermato-Venereologica 78:136138.

Lontz W, Olsson MJ, Moellmann G, and Lerner AB. 1994. Pigment cell transplantation for treatment of vitiligo: a progress report. Journal of the American Academy of Dermatology 30:591-597.

Mulekar SV. 2003. Melanocyte-keratinocyte cell transplantation for stable vitiligo. International Journal of Dermatology 42:132-136.

Naysmith L, Waterston K, Ha T, Flanagan N, Bisset Y, Ray A, Wakamatsu K, Ito S, and Rees JL. 2004. Quantitative measures of the effect of the melanocortin 1 receptor on human pigmentary status. J Invest Dermatol 122:423-428. 10.1046/j.0022-202X.2004.22221.X

Olsson MJ, and Juhlin L. 2002. Long-term follow-up of leucoderma patients treated with transplants of autologous cultured melanocytes, ultrathin epidermal sheets and basal cell layer suspension. British Journal of Dermatology 147:893-904.

Snell RS, and Bischitz PG. 1963. The Melanocytes and Melanin in Human Abdominal Wall Skin: A Survey Made at Different Ages in Both Sexes and during Pregnancy. Journal of Anatomy 97:361-376.

Staricco RJ, and Pinkus H. 1957. Quantitative and qualitative data on the pigment cells of adult human epidermis. Journal of Investigative Dermatology 28:33-45.

Stevenson JM, Weatherall IL, Litilejohn RP, and Seman DL. 2012. A comparison of two different instruments for measuring venison CIELAB values and colour assessment by a trained panel. New Zealand Journal of Agricultural Research 34:207-211. 10.1080/00288233.1991.10423361

Stokowski RP, Pant PV, Dadd T, Fereday A, Hinds DA, Jarman C, Filsell W, Ginger RS, Green MR, van der Ouderaa FJ, and Cox DR. 2007. A genomewide association study of skin pigmentation in a South Asian population. Am J Hum Genet 81:1119-1132. 
432

433

434

435

436

437

438

439

440

441

442

443

444

445

446

447

448

449

450

451

452

453

454

455

456

457

458

459

460

461

462

463

464

465

466

467

$10.1086 / 522235$

Swope VB, Supp AP, and Boyce ST. 2002. Regulation of cutaneous pigmentation by titration of human melanocytes in cultured skin substitutes grafted to athymic mice. Wound Repair and Regeneration 10:378-386. 10.1046/j.1524-475X.2002.10607.x

Swope VB, Supp AP, Cornelius JR, Babcock GF, and Boyce ST. 1997. Regulation of pigmentation in cultured skin substitutes by cytometric sorting of melanocytes and keratinocytes. $J$ Invest Dermatol 109:289-295.

Szabo G, Gerald AB, Pathak MA, and Fitzpatrick TB. 1969. Racial differences in the fate of melanosomes in human epidermis. Nature 222:1081-1082.

Thingnes J, Lavelle TJ, Hovig E, and Omholt SW. 2012. Understanding the melanocyte distribution in human epidermis: an agent-based computational model approach. PLoS One 7:e40377. 10.1371/journal.pone.0040377

Tsuchida Y, Fukuda O, and Kamata S. 1991. The correlation of skin blood flow with age, total cholesterol, hematocrit, blood pressure, and hemoglobin. Plastic and Reconstructive Surgery 88:844-850.

van Geel N, Ongenae K, De Mil M, and Naeyaert JM. 2001. Modified technique of autologous noncultured epidermal cell transplantation for repigmenting vitiligo: a pilot study. Dermatologic Surgery 27:873-876.

Wakamatsu K, Kavanagh R, Kadekaro AL, Terzieva S, Sturm RA, Leachman S, Abdel-Malek Z, and Ito $S .2006$. Diversity of pigmentation in cultured human melanocytes is due to differences in the type as well as quantity of melanin. Pigment Cell Research 19:154-162. 10.1111/j.1600-0749.2006.00293.x

Weatherall IL, and Coombs BD. 1992. Skin color measurements in terms of CIELAB color space values. Journal of Investigative Dermatology 99:468-473.

Wilson S, Ginger RS, Dadd T, Gunn D, Lim FL, Sawicka M, Sandel M, Schnetkamp PP, and Green MR. 2013. NCKX5, a natural regulator of human skin colour variation, regulates the expression of key pigment genes MC1R and alpha-MSH and alters cholesterol homeostasis in normal human melanocytes. Adv Exp Med Biol 961:95-107. 10.1007/9781-4614-4756-6_9

Yin L, Coelho SG, Ebsen D, Smuda C, Mahns A, Miller SA, Beer JZ, Kolbe L, and Hearing VJ. 2014. Epidermal gene expression and ethnic pigmentation variations among individuals of Asian, European and African ancestry. Experimental Dermatology 23:731-735. 10.1111/exd.12518 
468 Figure Legends

469 Fig. 1 The illustration of the process for measurement of skin color and formulation of the relation 470 among $L^{*}$ value, epidermal melanocyte ratio and melanin concentration.

471 Fig. 2 Determination of epidermal melanocyte ratio (100x; scale bar: $100 \mu \mathrm{m})$ (a) The foreskin 472 samples were treated immediately with the FACS method. The results showed up to $2.44 \%$ of 473 melanocytes (M1) in a human adult foreskin sample. (b) The results of fluorescent-activated cell 474 sorting (FACS) showed up to $99.4 \%$ purity of melanocytes (M2) in a selected PHEMs culture. (c) 475 Selected primary culture of human epidermal melanocytes (PHEMs) was shown in bright field. 476 (d) The PHEMs in selected primary culture were labeled by anti-melan antibody incorporated 477 with fluorescein isothiocyanate (FITC).

478 Fig. 3 Statistical correlations between skin color-space parameter: $L^{*}$ value and epidermal 479 melanocyte ratio or melanin concentration. (a) $L^{*}$ value corresponding to epidermal melanocyte 480 ratio expresses a random distribution where the relation between them seem to be fairly weak. 481 Correlation coefficient, $r=-0.081(P=0.774)$ and coefficient of determination, $R^{2}=0.0066$ (b) $A$ 482 slight trend of a higher $\mathrm{L}^{*}$ value corresponding to a higher melanin concentration per $10^{6}$ 483 melanocytes was observed. Correlation coefficient, $r=-0.592(P=0.02)$ and coefficient of 484 determination, $\mathrm{R}^{2}=0.3503$.

485 Fig. 4. Statistical correlations between skin color-space parameter: $L^{*}$ value and the product of 486 epidermal melanocyte ratio and melanin concentration. A strong correlation was shown between $487 L^{*}$ value and the value of epidermal melanocyte ratio $(R)(\%)$ multiplied by melanin concentration 488 (M) $\left(\mu \mathrm{g} / 10^{6}\right.$ melanocytes $)$. Correlation coefficient, $r=-0.847(P<0.001)$ and coefficient of 489 determination, $\mathrm{R}^{2}=0.717$.

490 Fig. 5. (a) The gross appearance of the collagen/PCL constructs at 70 days. (b) The L* value profiles 491 of the collagen/PCL constructs during a period of 70 days. The 6 -well tissue culture plastics 
492 without cells served as the blank group.

493 


\section{Table $\mathbf{1}$ (on next page)}

Patient profile of foreskin and demographic data ( $n=3$ for $L^{*}$ value and melanin concentration; $n=5$ for epidermal melanocyte ratio; Values are mean $\pm S D)^{1}$. 
1 Table 1 Patient profile of foreskin and demographic data ( $\mathrm{n}=3$ for $\mathrm{L}^{*}$ value and melanin concentration; $\mathrm{n}$

2 = 5 for epidermal melanocyte ratio; Values are mean $\pm \mathrm{SD})^{1}$.

\begin{tabular}{ccccc}
\hline $\begin{array}{c}\text { Samples } \\
\text { (No.) }\end{array}$ & $\begin{array}{c}\text { Age } \\
\text { (years) }\end{array}$ & $L^{*}$ value & $\begin{array}{c}\text { Melanin } \\
\text { Epidermal melanocyte }\end{array}$ & $\begin{array}{c}\text { ratio }(\%) \\
\text { concentration } \\
\left(\mu \mathrm{g} / 10^{6} \text { melanocytes) }\right.\end{array}$ \\
\hline 1 & 23 & $39.43 \pm 0.21$ & $1.96 \pm 0.09$ & $68.96 \pm 0.20$ \\
2 & 25 & $47.27 \pm 1.90$ & $3.18 \pm 0.24$ & $34.61 \pm 0.39$ \\
3 & 24 & $41.20 \pm 2.17$ & $3.13 \pm 0.10$ & $41.50 \pm 0.34$ \\
4 & 24 & $46.97 \pm 1.52$ & $1.40 \pm 0.28$ & $81.91 \pm 1.92$ \\
5 & 24 & $52.13 \pm 1.62$ & $1.97 \pm 0.31$ & $25.88 \pm 0.11$ \\
6 & 22 & $52.37 \pm 1.90$ & $1.44 \pm 0.09$ & $21.97 \pm 0.62$ \\
7 & 22 & $45.93 \pm 0.59$ & $2.70 \pm 0.10$ & $47.43 \pm 0.72$ \\
8 & 26 & $49.30 \pm 0.60$ & $3.49 \pm 0.14$ & $24.31 \pm 0.58$ \\
9 & 24 & $44.07 \pm 0.15$ & $2.44 \pm 0.10$ & $43.32 \pm 2.66$ \\
10 & 22 & $47.53 \pm 2.37$ & $2.71 \pm 0.52$ & $26.39 \pm 0.68$ \\
11 & 21 & $48.70 \pm 2.81$ & $3.40 \pm 0.22$ & $26.97 \pm 0.27$ \\
12 & 38 & $49.73 \pm 1.15$ & $3.58 \pm 0.07$ & $23.39 \pm 0.32$ \\
13 & 23 & $47.87 \pm 3.26$ & $3.23 \pm 0.22$ & $17.99 \pm 0.50$ \\
14 & 25 & $45.87 \pm 2.18$ & $1.68 \pm 0.12$ & $67.66 \pm 0.46$ \\
15 & 24 & $50.60 \pm 1.40$ & $1.41 \pm 0.14$ & $36.07 \pm 0.20$ \\
\hline
\end{tabular}

${ }^{1} n$, the number of tests performed on each individual sample. 


\section{Figure 1}

The illustration of the process for measurement of skin color and formulation of the relation among $L^{*}$ value, epidermal melanocyte ratio and melanin concentration.

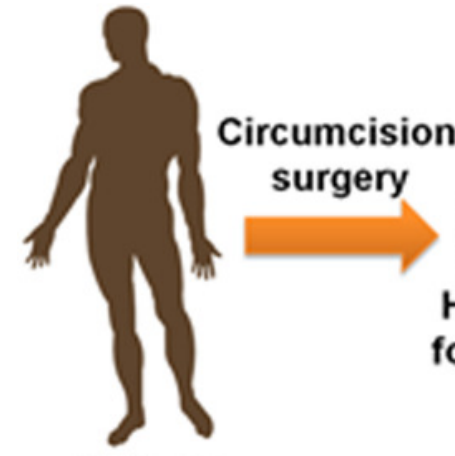

Patients

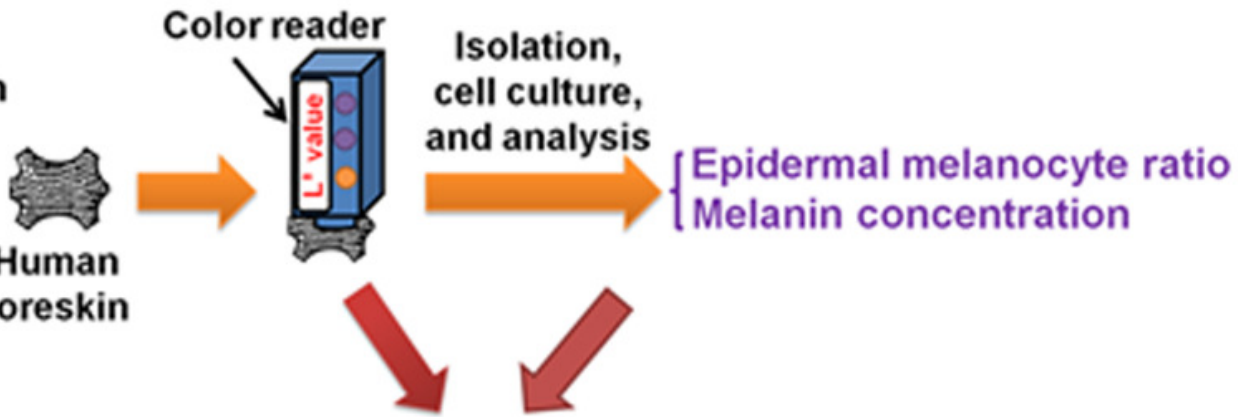

Color formula: $L^{*} \propto R$ \& $M$

$\left\{\begin{array}{l}L^{\prime} \text { value: skin color } \\ R \text { value: epidermal melanocyte ratio } \\ M \text { value: melanin concentration per } 10^{6} \text { melanocytes }\end{array}\right.$ 


\section{Figure 2}

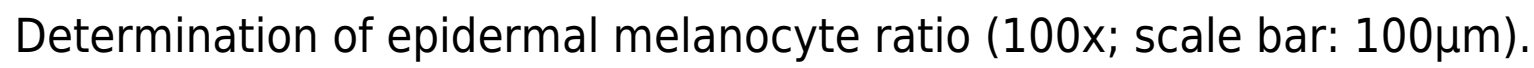

(a) The foreskin samples were treated immediately with the FACS method. The results showed up to $2.44 \%$ of melanocytes (M1) in a human adult foreskin sample. (b) The results of fluorescent-activated cell sorting (FACS) showed up to $99.4 \%$ purity of melanocytes (M2) in a selected PHEMs culture. (c) Selected primary culture of human epidermal melanocytes (PHEMs) was shown in bright field. (d) The PHEMs in selected primary culture were labeled by anti-melan antibody incorporated with fluorescein isothiocyanate (FITC). 
(a) PHEMs in a foreskin sample

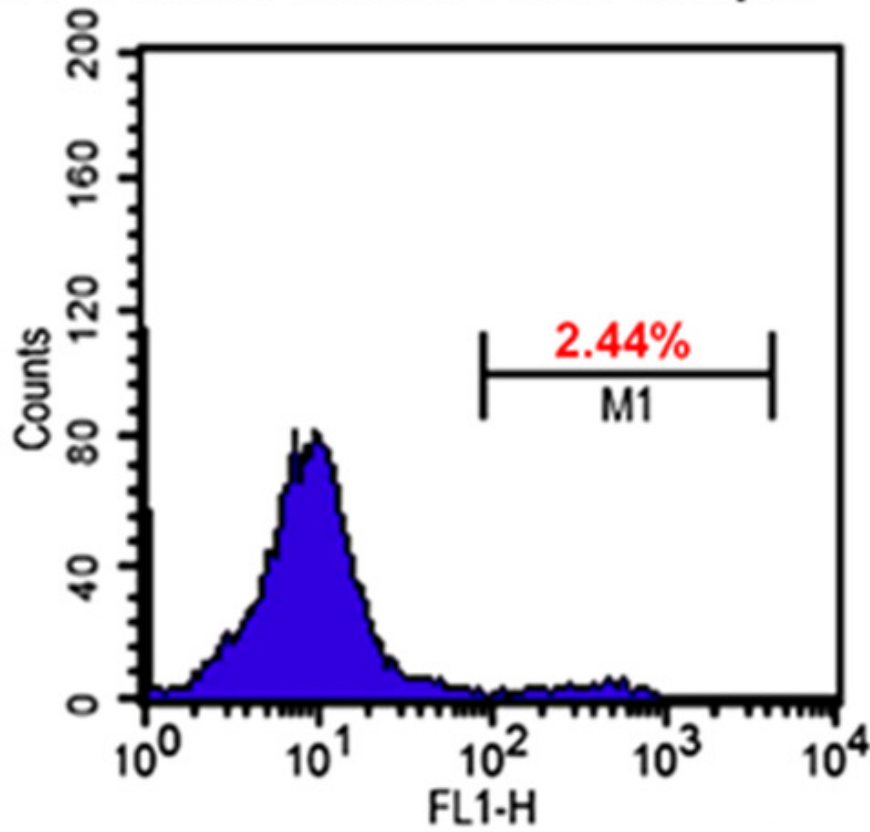

(c)

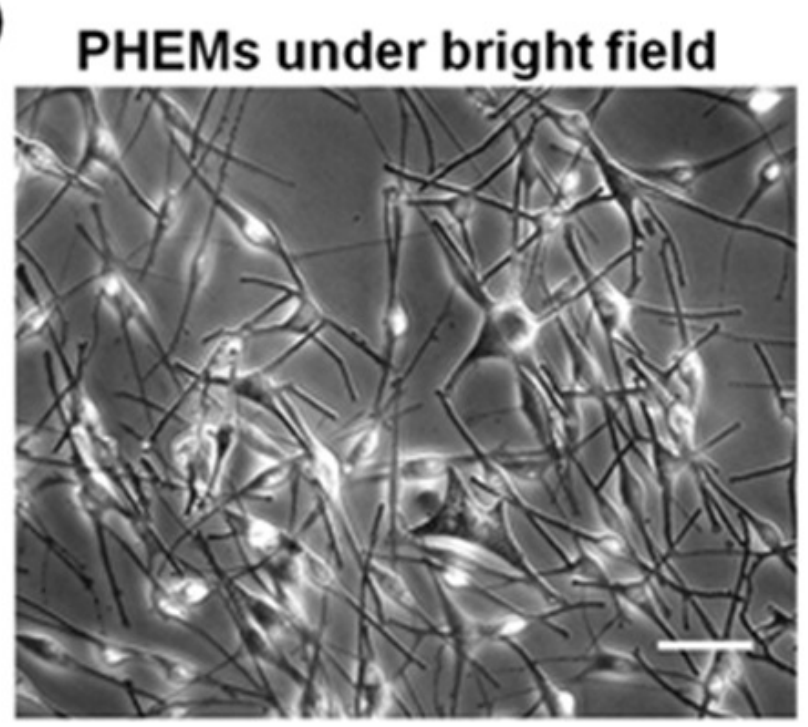

(b)

Purity of PHEMs

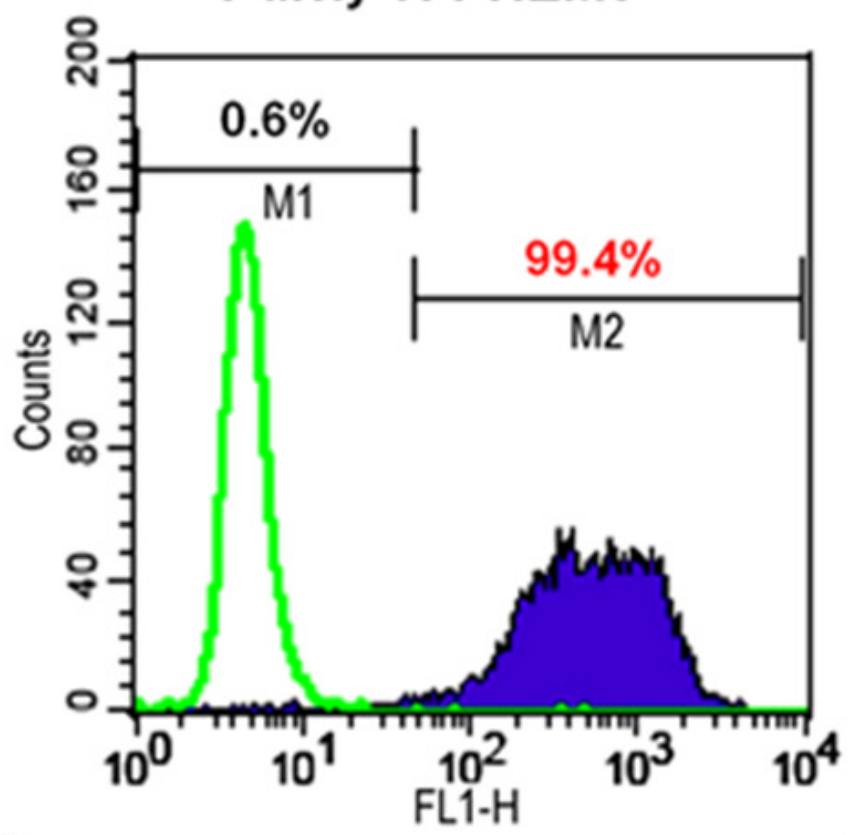

(d) PHEMs under FITC

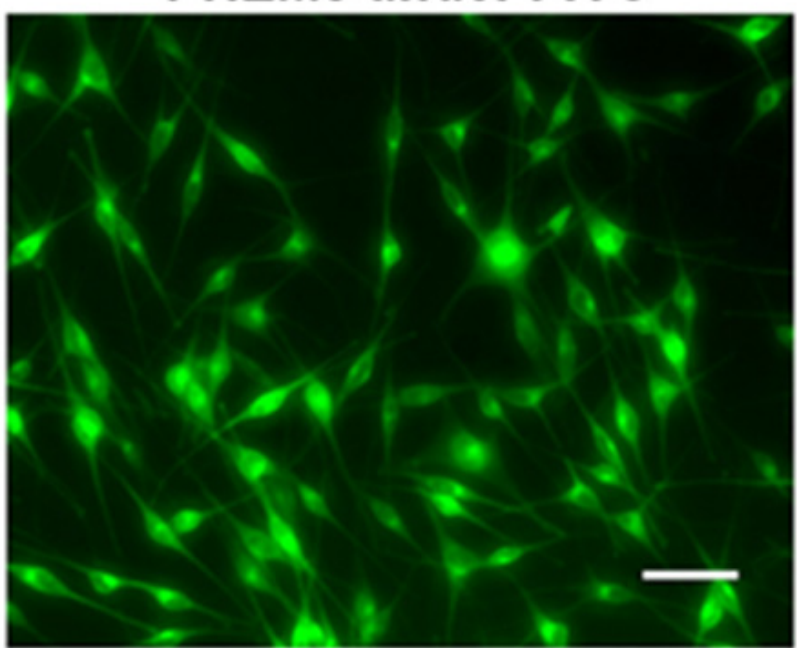


Figure 3

Statistical correlations between skin color-space parameter: $L^{*}$ value and epidermal melanocyte ratio or melanin concentration.

(a) $L^{*}$ value corresponding to epidermal melanocyte ratio expresses a random distribution where the relation between them seem to be fairly weak. Correlation coefficient, $r=-0.081$ $(P=0.774)$ and coefficient of determination, $\mathrm{R}^{2}=0.0066$ (b) A slight trend of a higher $\mathrm{L}^{*}$ value corresponding to a higher melanin concentration per 106 melanocytes was observed. Correlation coefficient, $r=-0.592(P=0.02)$ and coefficient of determination, $R^{2}=0.3503$.

(a)

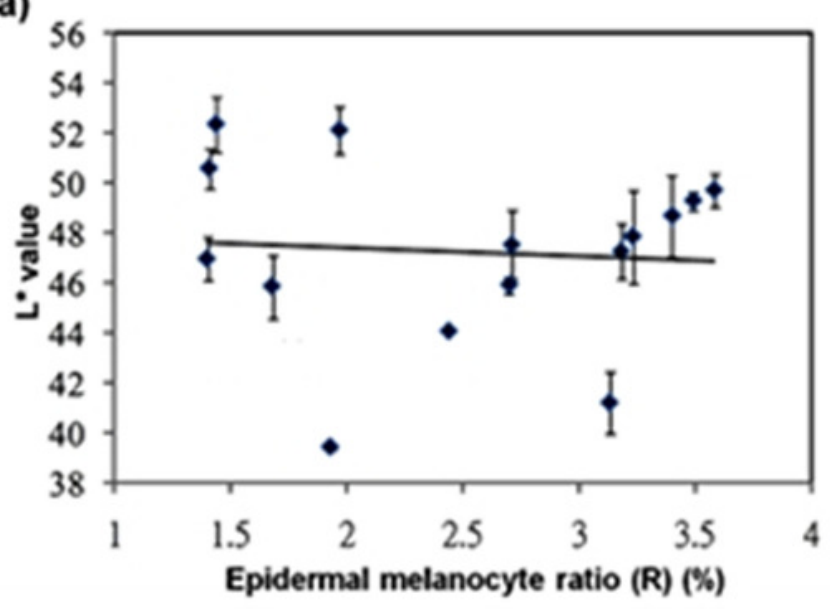

(b)

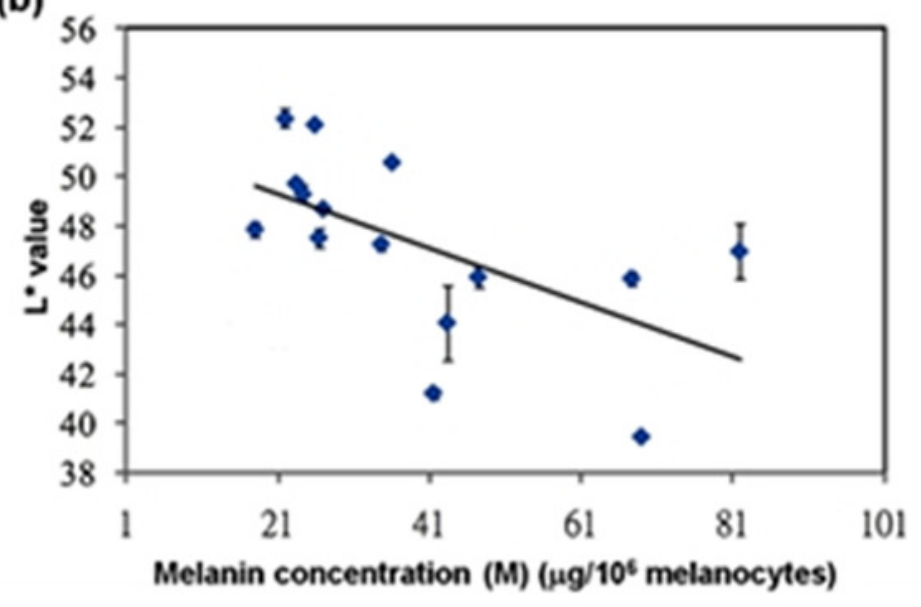


Figure 4

Statistical correlations between skin color-space parameter: $L^{*}$ value and the product of epidermal melanocyte ratio and melanin concentration.

A strong correlation was shown between $L^{*}$ value and the value of epidermal melanocyte ratio (R) (\%) multiplied by melanin concentration (M) ( $\mathrm{ug} / 10^{6}$ melanocytes). Correlation coefficient, $r=-0.847(P<0.001)$ and coefficient of determination, $R^{2}=0.717$.

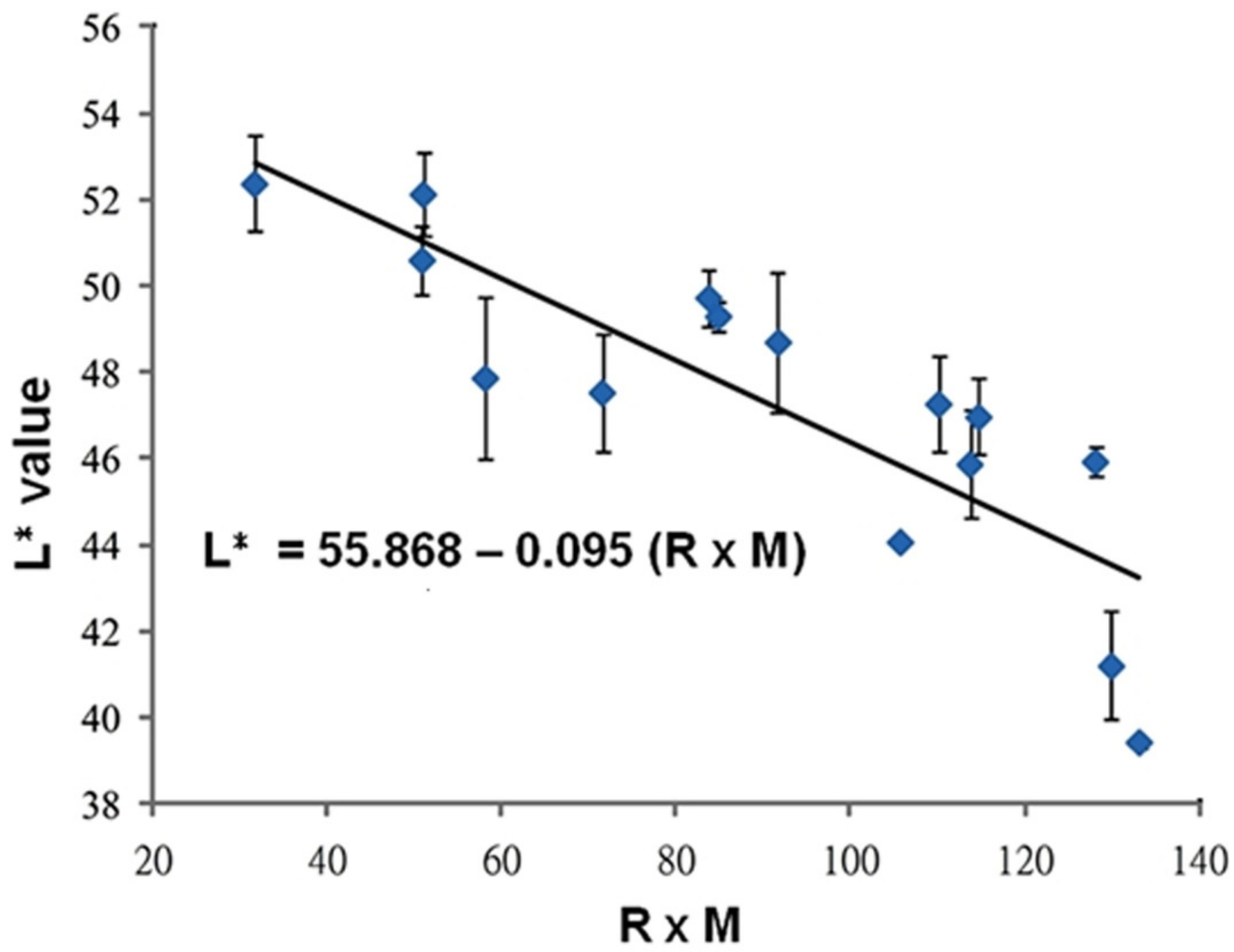


Figure 5

(a) The gross appearance of the collagen/PCL constructs at 70 days. (b) The L* value profiles of the collagen/PCL constructs during a period of 70 days. The 6 -well tissue culture plastics without cells served as the blank group. 
(a)
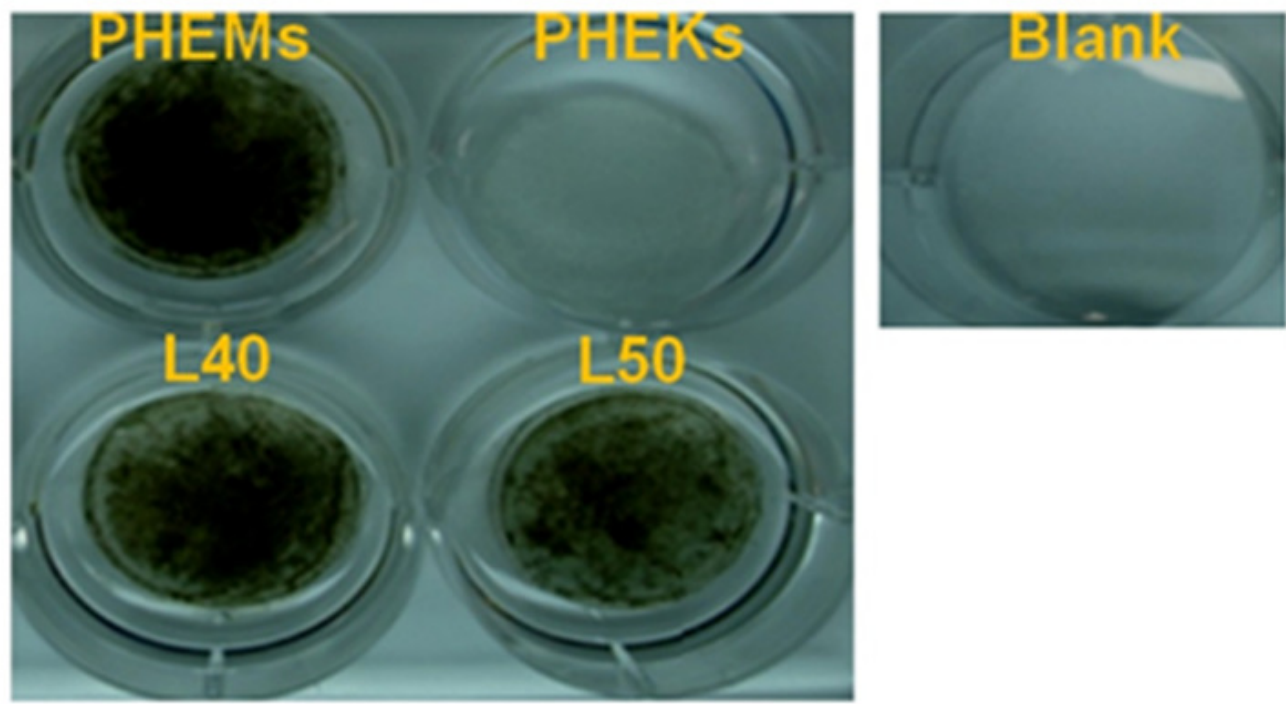

(b)

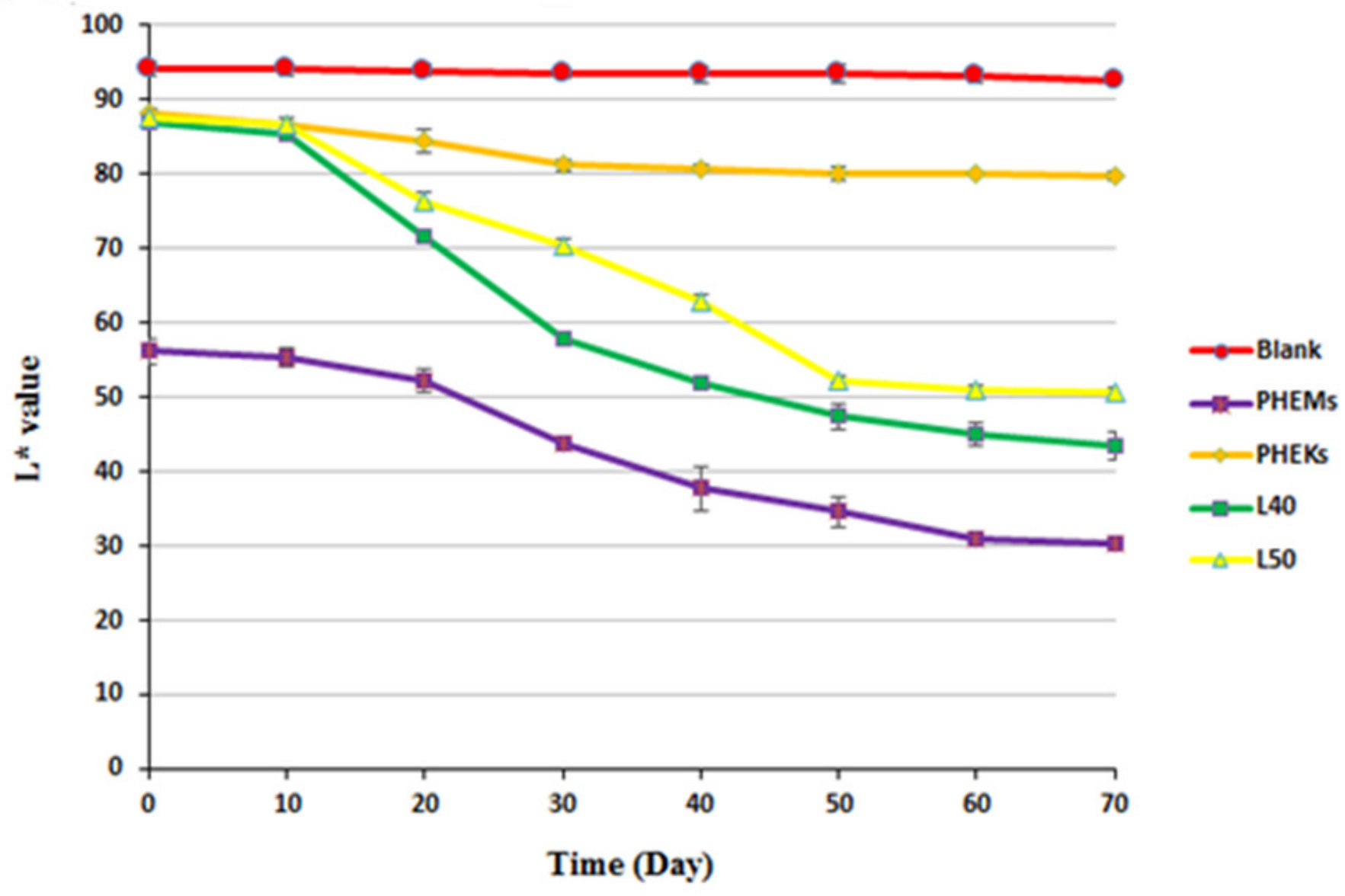

